\title{
Modeling and Parametric Study of the Heat Transfer Inside a Lithium Bromide Flow Confined by Wire Screen
}

\author{
Herbert Obame $\mathrm{Mve}^{1,2}$, Romuald Rullière ${ }^{2}$ and Philippe Haberschill ${ }^{2}$ \\ 1. Peugeot Société Anonyme Peugeot Citroën, Centre Technique de Vélizy, Vélizy Villacoublay Cedex 78943, France \\ 2. Centre National de la Recherche Scientifique, Institut National des Sciences Appliquées de Lyon, Centre d'Energétique et de \\ Thermique de Lyon, Villeurbanne Cedex 69621, France
}

Received: November 27, 2014 / Accepted: January 08, 2015 / Published: May 31, 2015.

\begin{abstract}
The present study deals with the numerical analysis of heat transfer inside a lithium bromide-water solution flowing down between finely meshed plastic wire screens. These screens confine the flow through capillary action while allowing the water vapour transfer inside an innovative absorber technology. The complex menisci shape formed on the confinement grid level, where the surface tension forces are of first importance, are reconstructed by a volume-of-fluid model. A continuum surface force model is used to account for the surface tension force. A static contact angle is used to define the wall adhesion. A new algorithm, consisting to set an unique constant temperature at the liquid/vapour interface and to determine the evolution of heat transfer characteristics over the simulation domain, has been implemented and validated by analytical solution. A parametric study has been conducted to determine the effect of the geometry, the contact angle and the shape of the wire on the heat transfer.
\end{abstract}

Key words: Heat transfer, wire screen, complex menisci, liquid/vapour interface, numerical simulation.

\section{Introduction}

The new European environmental regulation requires reducing greenhouse gas emissions and the use of harmful refrigerant. The air conditioning system implemented in private individual cars consists of a classical vapour compression cycle. The mechanical compression engenders a significant extra fuel consumption of heat engine vehicles. Besides, mobile air conditioning systems mainly use the refrigerant R134a whose GWP (global warning potential) exceeds 1,000 . R134a is gradually suppressed according to the European Directive 2006/40/EC EC (European Commission). The efficiency of mobile air conditioning systems will soon be constrained by an European regulation. Hence, the automotive manufacturers strengthen their efforts to make technology breakthroughs that would permit to face the

Corresponding author: Herbert Obame Mve, Ph.D., research fields: thermal and energetic. E-mail: mvhe07@hotmail.com. growing legal pressure. The absorption air conditioning system is a promising alternative as it may be driven by waste heat from the engine and uses zero-GWP fluids such as the water/lithium bromide pair. The classical evaporator/absorber where the fluids flow down over bundle of tubes is not suited to an automotive application because of the high risk of mixing of the fluids under the severe conditions encountered in a vehicle (inertia forces, vibrations, various orientations from the horizontal). Hence, we have developed an innovative evaporator/absorber where the fluids flow down between finely meshed wires screens that confine the flow through capillary action while allowing the vapour transfer. Previous investigations [1] have shown that, this innovative component is robust enough to support the severe automotive constraints but need improvement to be implemented in an automotive under hood environment. The optimization of the system requires investigating the complex hydrodynamics between the 
finely meshed plastic wire screens. The capillary effects are of first importance. The menisci that form on the three dimensional geometry constitute the transfer surface. Their shape results from a coupling between the flow and surface energy effects.

Several numerical studies for flow over the surface have been performed to investigate the effect of heat and mass transfer across deformed or stable interfaces [2, 3] and especially in the case of vapour absorption by the lithium bromide-water solution. The volume of fluid method [4] has been used to study heat and mass transfer numerically in simple cases as spherical droplets or bubbles. However, there are no reported studies of more complex flows that are typically encountered in the wire screen.

In this work, we focus on the heat transfer across the interface formed by menisci resulting from the flow confined by a woven wire screen. Because of the complexity of the geometry, the topology of the flow changes rapidly with time, making simulations difficult. Moreover, the complex nature of the menisci complicates the estimation of the heat flux normal to the interface. A new algorithm has been developed and implemented. This algorithm sets an unique temperature in the cells containing the interface and follows the evolution of the temperature in whole of the computational domain. The validation of the model has been performed by confrontation with analytical solutions in a simple case of diffusion process in one-dimensional heat transfer. A parametric study was done to determinate the effect of the contact angle, the inlet lithium bromide velocity, and the shape of the wire diameter on the transfer. The problem is simulated using the VOF (volume of fluid) model proposed by the computational fluid dynamics software FLOW-3D ${ }^{\circledR}$ in which the VOF method is combined to the FAVOR (fractional area/volume obstacle representation), a porosity technique used to define obstacles or solid [5].

\section{Numerical Simulation}

The simulation performs the heat transfer by setting an unique value of the temperature at the interface formed by complex menisci. These menisci are tracked using volume of fluid model [4]. As shown in Fig. 1a, the simulated domain can be divided in three parts:

Part 1: a closed microchannel of $5 \mathrm{~mm}$ length, sufficient to have an established flow before the grid;

Part 2: the grid (wire screen) of $5.52 \mathrm{~mm}$ length, principal part of the computational domain;

Part 3: an open microchannel of $1.7 \mathrm{~mm}$ length.

The domain length, width and height are respectively equal to $L_{c}=12.22 \mathrm{~mm}, l_{c}=1.38 \mathrm{~mm}$ and $h_{c}=0.5 \mathrm{~mm}$. The grid length $L_{g}$ is equal to $5 \mathrm{~mm}$ and the grid width $l_{g}$ is equal to the domain width $l_{c}$. The grid consists of a succession of meshes inside which the menisci are formed. This one is characterized by the wire diameter $d_{w}$ and the distance between two adjacent wires $L_{w}$ as shown in Fig. 1b. The bottom grid position is equal to channel height. The geometry is also characterized by the porosity $\varepsilon$ (Eq. (1)) that represents the total surface of the openings in the wire screens. Fig. 1c shows the 3D representation of the geometry:

$$
\varepsilon=\left[\frac{L_{w}}{L_{w}+d_{w}}\right]^{2}
$$

\subsection{Assumption and Model Formulation}

In this study, heat transfer is assumed to have no effect on the volume fraction of flow in each computational cell. The interface is assumed to have no resistance to diffusion, so that equilibrium conditions apply at any point of the interface where a unique temperature is set. Also as a consequence of one phase consideration, we assume that, the vapour pressure is constant and uniform and the viscous stress at the liquid/vapour interface is negligible. The governing equations for the VOF model, for an incompressible flow, in Cartesian coordinates, without source term, in the one fluid formulation takes the following form:

$$
\nabla \cdot(U \chi)=0
$$

$\frac{\partial U}{\partial t}+\frac{1}{\beta_{f}} \nabla \cdot(\rho U U \chi)=-\nabla P+\nabla \cdot \tau+\rho g+F_{S}$

$$
\beta_{f} \frac{\partial f}{\partial t}+\nabla \cdot(U \chi f)=0
$$




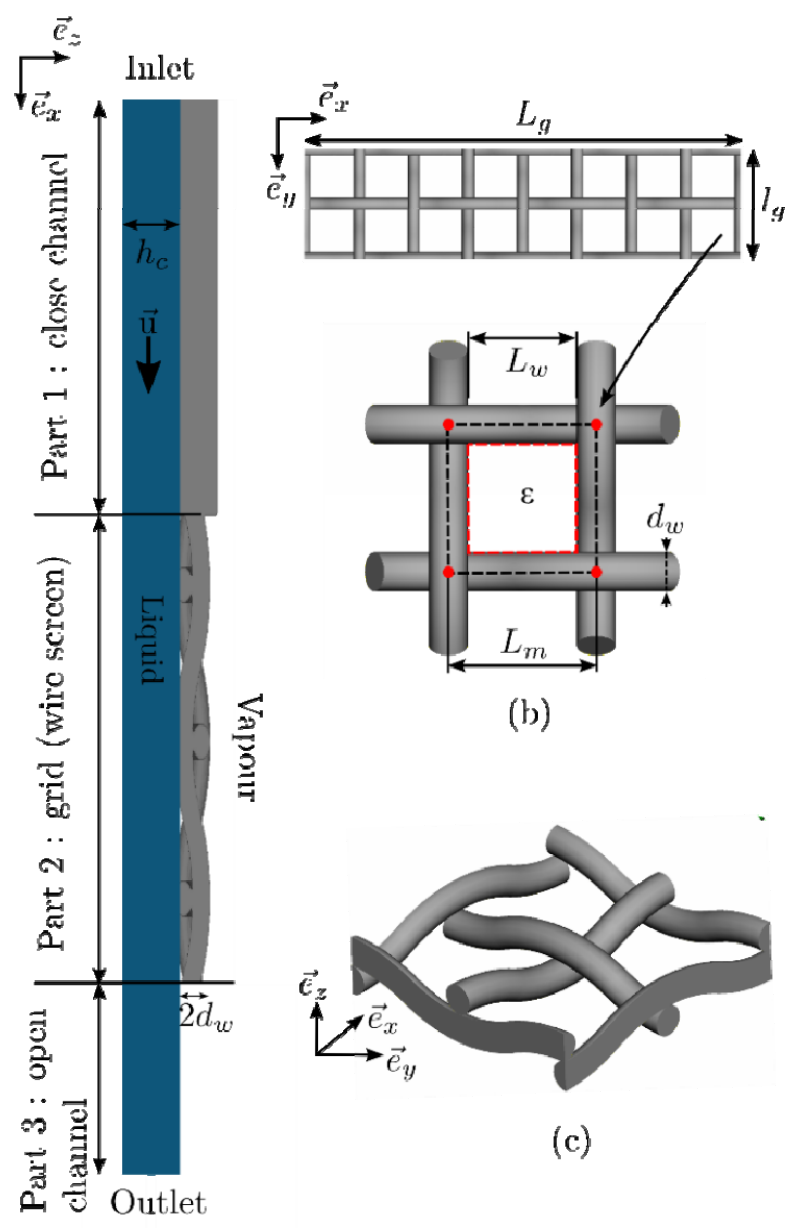

(a)

Fig. 1 (a) Description of the simulated; (b) domain description of the geometry simulated; (c) 3D visualization of the wire screen.

$$
\beta_{f} \rho C_{p} \frac{\partial T}{\partial t}+\rho C_{p} \nabla \cdot(U \chi T)=\nabla \cdot(\lambda \chi \nabla T)
$$

Where $U$ is the velocity, $\chi$ is the fractional area open to flow, $\beta_{f}$ is the fractional volume open to flow, $\rho$ is the density, $P$ is the pressure, $\tau=\mu\left(\nabla U+\nabla U^{T}\right)$ denotes the viscous stress tensor, with $\mu$ representing the dynamic viscosity, $g$ and $F_{S}$ are gravity acceleration and surface tension forces, respectively, $f$ is the liquid fraction and $f$ varies between 0 and $1: f=0$ in void cells, $f=1$ in cells filled with liquid and $0<f<1$ in cells that contain interface. $C_{p}$ is the specific heat, $T$ is the temperature and $k$ is the thermal conductivity. In Eq. (5), the heat transfer between solid and liquid is not taken into account.

As said before, the temperature is set at the center of the cell that contains the interface. The advection of the temperature depends on the liquid fraction $f$ as a consequence that the heat flux depends on the $f$ value in each interface computational cell. This dependency caused numerical errors, because the flux is underestimated in the cells that contain the interface with small $f$ values. To reduce this error, a scalar $\varphi$ is introduced to set the temperature in the cell $z_{i}$ that contains the interface and in the adjacent cell $z_{i-1}$ or only in the interface cell as:

(1) For $f \leq \varphi, T_{i}$ is set in both cells $z_{i}$ and $z_{i-1}$;

(2) For $f>\varphi, T_{i}$ is set only in the cell that contains interface $z_{i}$.

Where, $T_{i}$ is the interface temperature, $\varphi$ is funded numerically and $\varphi=0.325$.

\subsection{Boundary Conditions}

At the inlet of the domain, the $x$ velocity $u_{0}$ is set and the $y$ and $z$ velocity components are equal to zero. At the outlet, all the gradients are set to zero. At the walls, a no-slip boundary condition is applied, where all the normal velocity components are equal to zero. At the interface, it is assumed that, the pressure exerted by the vapour phase on the liquid phase is constant and uniform, equals to the atmospheric pressure, it constitutes the liquid phase boundary condition at the liquid/vapour interface.

\subsection{Numerical Treatment}

The conservation equation for the liquid fraction $f$ (Eq. (4)) is solved implicitly by means of a first order Lagrangian advection scheme [6]. The interface reconstruction is realized through the PLIC (piecewise linear interface calculation) [7]. The wall adhesion is taken into account through an unique contact angle. The treatment of the momentum equation is fully implicit, and the surface tension model [8] is solved implicitly to avoid problem of time limitation. The SIMPLEC (semi-implicit method for pressure-linked equation consistent) method is used for the pressure velocity coupling. 
A first simulation is realized with a coarse grid, with about 30,000 cells, in order to speed up the convergence and to reach the stationary state in a reasonable computing time. The second simulation is run from this state with a finer grid, about 5,000,000 cells, in order to get an accurate solution. The last simulation is made from the second simulation to estimated heat transfer. In this simulation, the momentum equation is not solved to avoid problem of instability due to surface tension and to increase the convergence of the simulation. For all simulations, a uniform mesh size is used in the part containing the wire screen and non-uniform mesh otherwise, in $x$ direction. In both directions y and $z$, the mesh size is uniform and equal to the minimum mesh size in the $\mathrm{x}$ direction.

\subsection{Initial Conditions}

Initially, at $t=0 \mathrm{~s}$, the free surface elevation of the liquid in the microchannel is set to $1.0 \mathrm{~mm}$ and the inlet velocity is set to $0.20 \mathrm{~m} / \mathrm{s}$. The liquid pressure is equal to $10^{5} \mathrm{~Pa}$ and the inlet temperature is equal to $305 \mathrm{~K}$. The interface temperature is equal to $370 \mathrm{~K}$ (equal to the water saturation temperature at the atmospheric pressure). The initial concentration of lithium bromide water solution is equal to 0.6 .

\section{Results and Discussion}

In this works, only the heat transfer is considered. The mass transfer is not taken into account as the consequence there are no coupling between heat and mass transfer. Table 1 summarized the simulation parameters used in this study $[9,10]$.

\subsection{Model Validation and Heat Flux Extrapolation}

The validation has been made by confronting numerical results with analytical solutions considering only the heat conduction. In this case, the problem can be reduced to a one-dimensional formulation. The analytical solution in this case is easily obtained in the dimensionless form proposed by Grossman [11]:
Table 1 Simulation parameters for a lithium bromide solution with a concentration of 0.6 .

\begin{tabular}{lll}
\hline Parameters & Values & Units \\
\hline$\rho$ & 1,734 & $\mathrm{~kg} / \mathrm{m}^{3}$ \\
$\lambda$ & 0.4122 & $\mathrm{~W} /(\mathrm{m} \cdot \mathrm{k})$ \\
$C_{p}$ & 1,928 & $\mathrm{~J} / \mathrm{kg}$ \\
$\mu$ & 0.0065 & $\mathrm{~Pa} \cdot \mathrm{s}$ \\
$\sigma$ & 0.0095 & $\mathrm{~N} / \mathrm{m}$ \\
$\alpha$ & $1.233 \times 10^{-7}$ & $\mathrm{~m}^{2} / \mathrm{s}$ \\
\hline & & \\
& $T^{*}=\frac{T_{(Z, t)}-T_{i}}{T_{0}-T_{i}}=\left[1-\operatorname{erf}\left(\frac{Z}{2 \cdot \sqrt{\alpha t}}\right)\right]$
\end{tabular}

With, $T_{(z, 0)}=T_{0}=303 \mathrm{~K}, T_{0}$ is the initial temperature, $T_{(0, t)}=T_{i}=373 \mathrm{~K}$, erf is the gauss error function and $a=\frac{\lambda}{\rho C_{p}}$ is the thermal diffusivity.

Fig. 2a shows a good agreement between the numerical and analytical solutions. The dimensionless absolute error $\Delta T$ is about $10^{-3}$. This one decreases with time as shown in Fig. $2 \mathrm{~b}$ because of, numerically, the steady state has not been reached.

The heat flux $\phi$ that diffuses across the interface (Eq. (7)) is the difference of two heat rate divides by the distance $\Delta L_{g}$ and the channel width $l_{g}$ as shown in Fig. 3. At each plane, $P_{1}$ and $P_{2}$, the heat rate increases rapidly attempting the maximum corresponding to the thermal steady state (Fig. 3). This one increases with the wire screen length explaining that $\phi_{2}$ is always superior to $\phi_{1}$ and $\phi$ is always positive.

$$
\begin{gathered}
\phi=\frac{1}{\Delta L_{g} l_{g}}\left(\phi_{2}-\phi_{1}\right)= \\
\frac{1}{\Delta L_{g} l_{g}} \int_{0}^{l_{g}} \int_{0}^{\delta} \rho c_{p}\left(u_{2} T_{2}-u_{1} T_{1}\right) \mathrm{d} s
\end{gathered}
$$

\subsection{Effect of the Wire Screen on the Heat Transfer}

Because of the special geometry of the wire screens, different modes of the heat transfer appear allowing the zone. In fact, two zones appear depending of the value of $\varepsilon$ and the position of the grid. At low porosity, where $\varepsilon$ is approximately less than or equal to 0.7 and $z$ superior to $h_{c}$, the flow is confined by wire screens and the capillary forces are of the first importance. In this region, the velocity is low and the average velocity is 


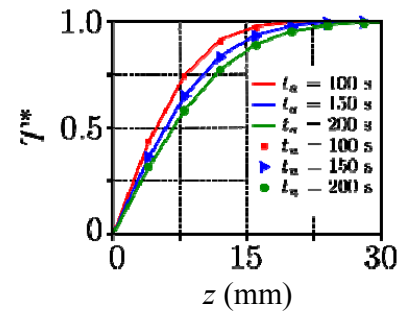

(a)

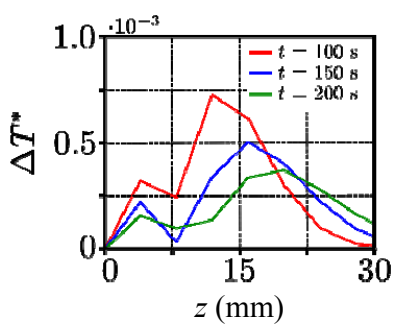

(b)
Fig. 2 Model validation: (a) evolution of dimensionless temperature (the subscript $\boldsymbol{a}$ and $\boldsymbol{n}$ indicates analytical and numerical solution, respectively); (b) the dimensionless absolute error.

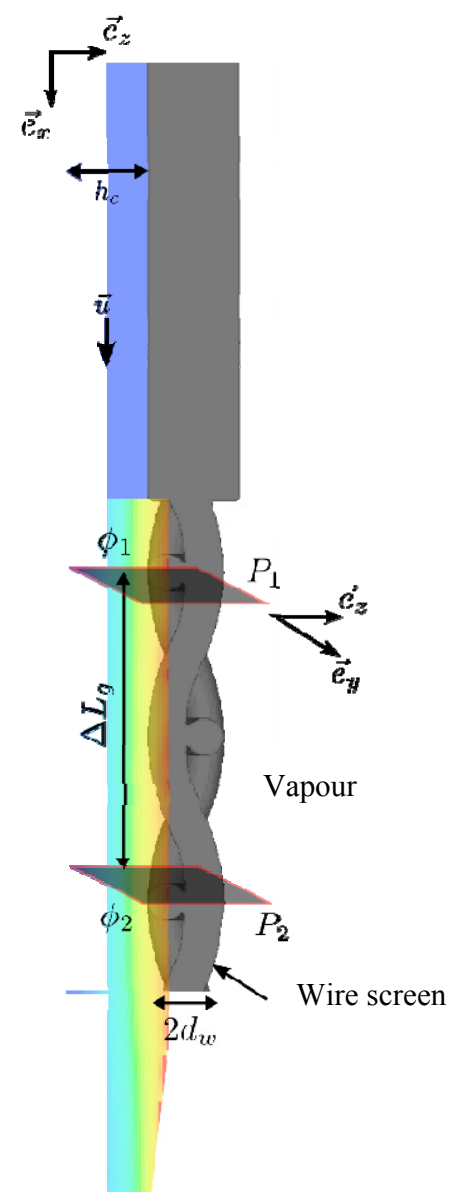

Fig. 3 Simulation domain representation.

equal of about $2 \mathrm{~mm} / \mathrm{s}$. The heat transfer is so a competition between the diffusion and convection as shown in Fig. 4a. The minimum and the maximum Peclet number are respectively 0 (close to the interface) and 5 for $x / L_{w}=0.5$. For $z \leq h_{c}$, the region under the wire screen, the flow is similarly to that one shown in the case of Poiseuille flow between two parallel plates. A fully $2 \mathrm{D}$ parabolic velocity is developed. The average velocity is 10-100 higher (centre of channel) than the velocity in the region containing the wire screen. In this case, the convection is dominant and the heat transfer due to convection is clearly dominant. This observation shows that, if the wire screen diameter increases and the porosity decreases, the zone $z>h_{c}$ increases reducing the heat flux across the interface because of convection is low or zero.

At high porosity where $\varepsilon$ is approximately more than 0.75 , there is no typical difference between of both zones. Velocity influences the heat transfer near to the interface and the heat transfer by convection is dominant as shown in Fig. $4 \mathrm{~b}$ that the minimum Peclet number is equal to 5 for $z>h_{c}$. As in the case for low porosity, the flow for $z \leq h_{c}$ is similary to this one in the case of poiseuille flow between two parallel plates.

Also, another effect that is important in the heat transfer with wire screen is the development of the vortex $\omega_{y}^{*}$ (Eq. (8)) in the flow due to the waving of the wire (Fig. 5). As shown in Fig. 5a, the vortices appear near to the wire in the direction of flow. These vortices increase the heat flux, by increasing the temperature diffusion across the interface (Fig. 5b).

These observations show that, generally in the case of the wire screen, interface flow is stabilised by surface tension reducing the heat flux across the interface. This effect increases when the open surface to flow decreases and/or when the wire diameter increases.

$$
\omega_{y}^{*}=\frac{\partial u^{*}}{\partial \mathrm{z}^{*}}-\frac{\partial w^{*}}{\partial x^{*}}
$$

where, $u^{*}$ and $w^{*}$ are the dimensionless velocity in the $\mathrm{x}$,

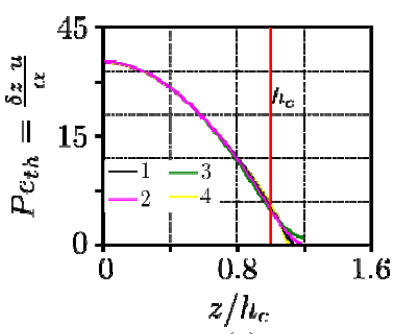

(a)

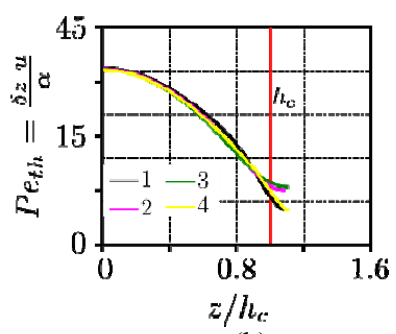

(b)
Fig. 4 Effect of the geometry, $1-x / L_{w}=0.25,2-x / L w=0.5$, $3-x / L_{w}=0.75,4-x / L_{w}=1$ : (a) Peclet number for $R e_{d w}=8.7$ and $\varepsilon=0.54$; (b) Peclet number for $R e_{d w}=4.7$ and $\varepsilon=0.82$. 
and $\mathrm{z}$ direction, respectively, $z^{*}$ and $x^{*}$ are the dimensionless axis.

\subsection{Effect of the Contact Angle}

The effect of contact angle has been performed with the wire diameter $d_{w}$ equal to $61 \mu \mathrm{m}$ and the distance between two adjacent wires $L_{w}$ equal to $629 \mu \mathrm{m}$. In this case, the porosity $\varepsilon$ is equal to 0.82 . The inlet velocity is equal to $0.288 \mathrm{~m} / \mathrm{s}$ and the range of the contact angle varies between $60^{\circ}$ and $95^{\circ}$.

Fig. 6a shows that, the heat flux increases rapidly from $52.25 \mathrm{~W} / \mathrm{cm}^{2}$ to about $54.5 \mathrm{~W} / \mathrm{cm}^{2}$ up to a contact angle equal to $85^{\circ}$. Beyond this value, the heat flux is constant whatever the static contact imposed. In Fig. 6a, the dimensionless heat transfer surface presents the same evolution to that one presented by the heat flux. In fact, the dimensionless heat transfer surface $G_{S}$ increases rapidly from $0.5 \%$ to $0.85 \%$ by meniscus, and thus remains constant. As shown in Fig. 1c, the wire screen is a 3D geometry and the meniscus resulting have a special shape describes experimentally by Mve, et al. [12]. This special shape constitutes the net surface transfer. This work shows that, the shape of
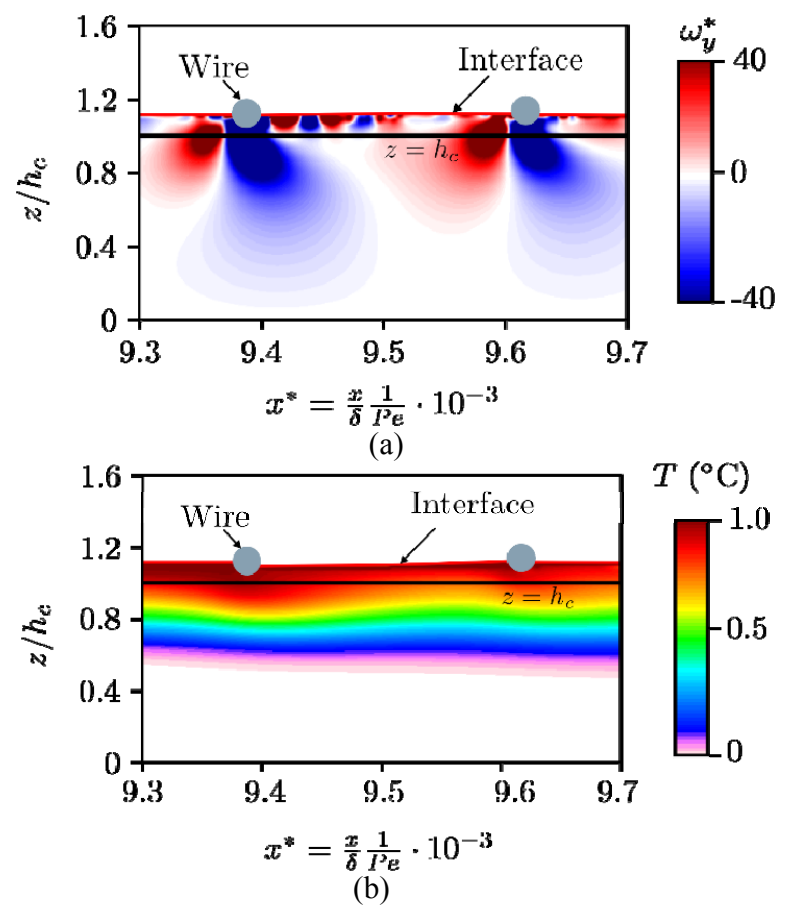

Fig. 5 (a) Temperature evolution and (b) vorticity zones at $y / L_{w}=0.5$ for $\operatorname{Red}_{w}=4.7$ and $\varepsilon=0.82$. the meniscus depends of the capillary forces and the three dimensional geometry.

Fig. 7 shows the different shapes of the meniscus near to the inlet of the computational domain $\left(x / L_{g} \approx\right.$ $0.25)$. It appears that, the free surface elevation is higher for a meniscus with low contact angle as shown in Fig. 6b, where the volume of fluid decreases with contact angle. This observation has been reported in many studies [13, 14]. Fig. 7 also shows that, as the contact angle increase, the geometry influence more the shape of the meniscus giving menisci with more curvature that increase the heat transfer surface. For a contact equal to $60^{\circ}$ until $80^{\circ}$, the shape of the meniscus near the inlet and the outlet is practically the same, with a curvature practically equal to zero at the center of the meniscus. For a contact angle equal or superior to $85^{\circ}$, between the inlet and the outlet of the computational domain, the shape of the meniscus change completely, with menisci having a convex curvature at the center, near the inlet, for menisci

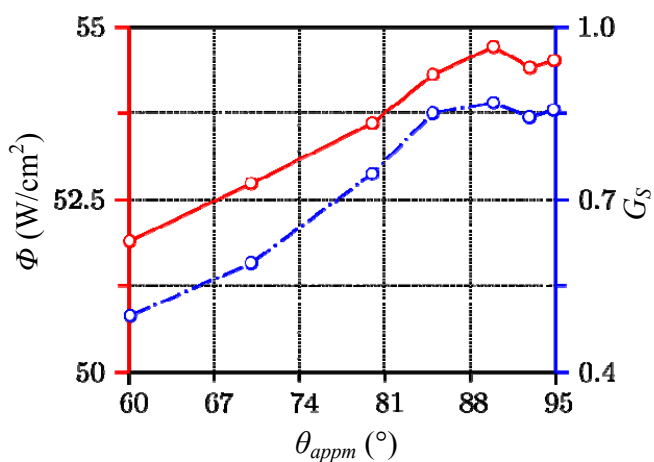

(a)

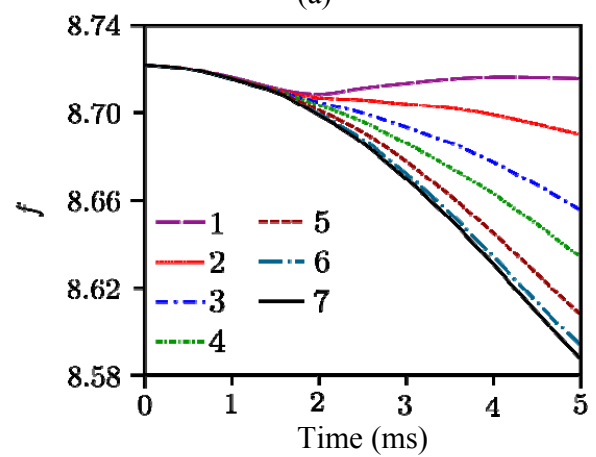

(b)

Fig. 6 Influence of the contact angle: (a) heat flux and dimensionless heat transfer surface; (b) volume of fluid with $1-\theta_{\text {appm }}=60^{\circ}, 2-\theta_{\text {appm }}=70^{\circ}, 3-\theta_{\text {appm }}=80^{\circ}, 4-\theta_{\text {appm }}=85^{\circ}$, $5-\theta_{\text {appm }}=90^{\circ}, 6-\theta_{\text {appm }}=93^{\circ}, 7-\theta_{\text {appm }}=95^{\circ}$. 

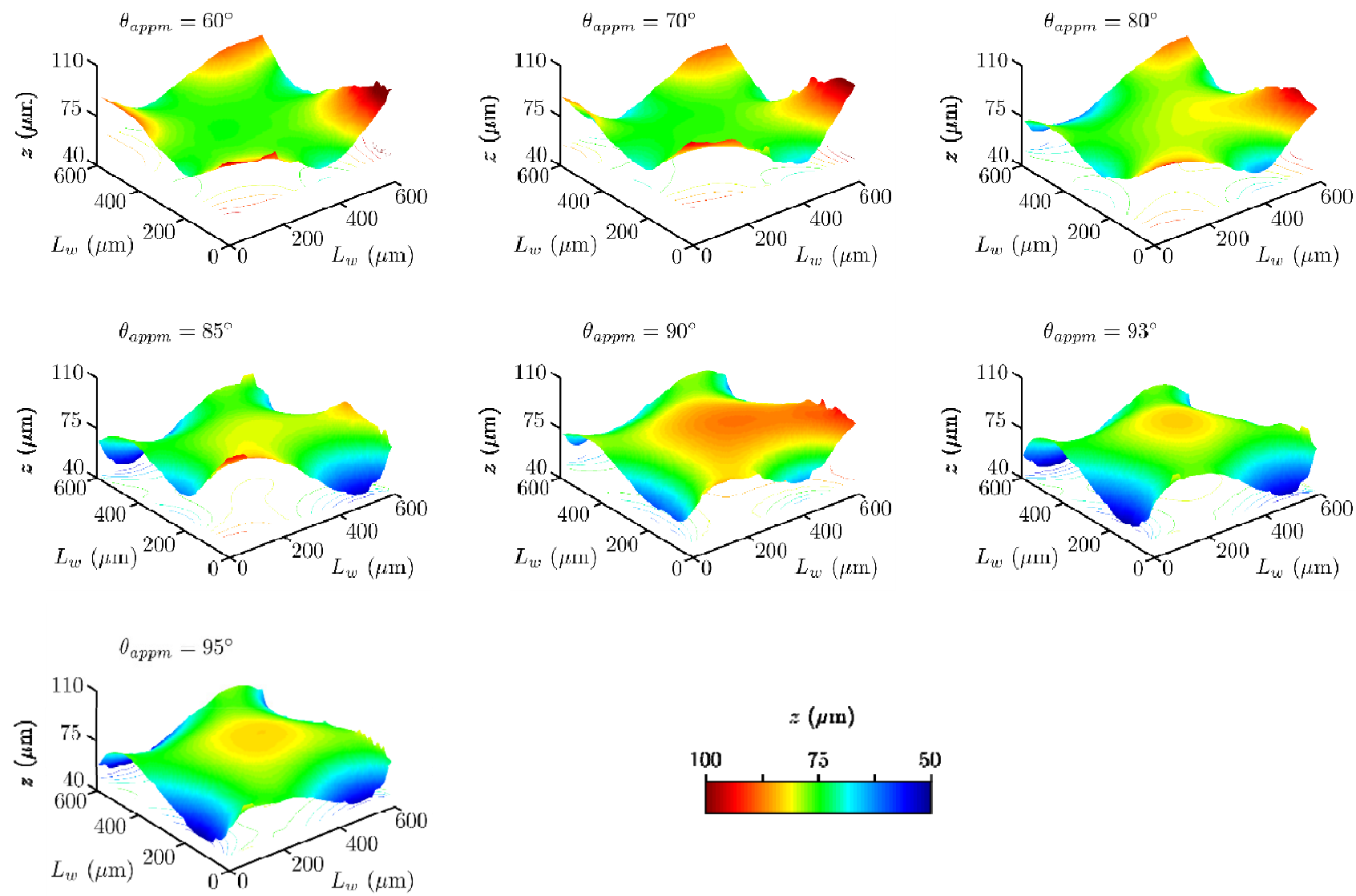

Fig. 7 Influence of the contact angle on the shape of the meniscus at the inlet of the wire screens.

having a concave curvature near the outlet. This evolution of the shape of menisci resulting by the balance between capillary forces and geometry explain the evolution of the dimensionless of the heat surface transfer with the static contact angle $\theta_{\text {appm }}$.

\subsection{Effect of the Shape of the Wire}

This part shows the comparison between the heat rate in the wire screens with circular wires and the heat rate with square wires in the same configuration described in Fig. 1. The simulation has been performed in the same conditions with the inlet velocity equal to $0.29 \mathrm{~m} / \mathrm{s}$ and the $d_{w}=61 \mu \mathrm{m}$ and the same porosity. The static contact angle is set to $90^{\circ}$.

The heat flux on the circular wire is partially $5 \%$ bigger than the one on the square wire. Regarding the shape of the meniscus, it appears that, in the case of the square wire, the waving have no great influence on the shape of the menisci (Fig. 8a). Menisci are practically a flat shape and the dimensionless surface regarding the surface projected is equal to $0.17 \%$ by meniscus. For the circular wire (Fig. 8b), and as shown in Section 3.3,

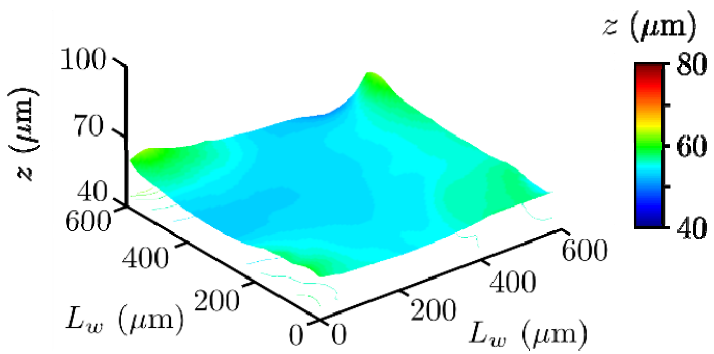

(a)

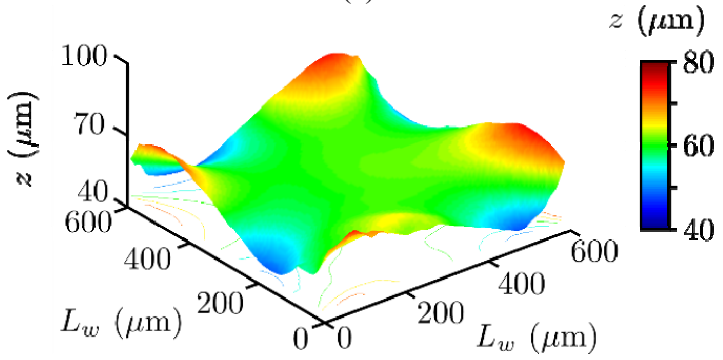

(b)

Fig. 8 Influence of the shape of the wire: (a) meniscus shape for square wire; (b) meniscus shape for circular wire. 
the waving influences more the shape of the meniscus. The average dimensionless heat transfer surface in this case is equal to $0.87 \%$ by meniscus. This result confirms the one in the previous section showing that, the heat flux depends on the heat transfer surface.

\section{Conclusions}

In summary, this work shows the influence of the geometry, contact angle and the shape of the wire on the heat transfer in the case of lithium bromide flow confined by wire screen.

It appears that, for low porosity, both zones can be distinguished depending of the position of the channel height $h_{c}$. For $z>h_{c}$, there are a competition between the heat transfer by convection and the heat transfer by diffusion. For $z \leq h_{c}$, the convection is dominant. At high porosity, there is no-difference between both zones. The velocity influences the heat transfer as well close to the interface and in the film. In this configuration, the heat transfer by convection is dominant. If the porosity decreases and the wire diameter increases, this configuration reduces strongly the heat transfer across the interface because of the increase of the zone with low convection $\left(z>h_{c}\right)$.

Also the effect of the static contact shows that, the shape of the meniscus is more and more independent of the geometry as the contact angle is low, reducing the heat transfer surface and thus the heat flux across the interface. For a contact angle superior or equal to $85^{\circ}$, the geometry influences more the shape of the meniscus and the heat transfer increases. In the configuration presented in this work, it is preferable to work for the contact angle between $85^{\circ}$ and $95^{\circ}$.

Finally, the comparison between the square wires and the circular wires reveals that, the circular wires are the best case. In fact, for circular wires the geometry influences more the shape of the menisci than in the square wires where the meniscus presents a flat shape. The heat flux is about $5 \%$ less than the one in the circular wires. This observation shows that, the circular wire ameliorate the heat flux that diffuse across the interface that square wires.

\section{Acknowledgments}

This work has been carried out in the framework of a collaboration between the CETHIL (Centre d'Energie et de Thermique de Lyon) and Peugeot Société Anonyme Peugeot Citroën. The authors gratefully acknowledge Flow Science and XC Engineering for the support on the simulations conducted.

\section{References}

[1] Boudard, E., and Bruzzo, V. 2004. Exchange Device and Heat Transfer Especially for Automotive Vehicle. French Patent 2,871,221, filed February 6, 2004, and issued December 9, 2005.

[2] Davidson, M. R. 2002. "Volume-of-Fluid Calculation of Heat or Mass Transfert across Deforming Interfaces in Two-Fluid Flow." Numerical Heat Transfert 41 (3-4): 291-308.

[3] Banerjee, R. 2007. "A Numerical Study of Combined Heat and Mass Transfer in Inclined Channel Using the VOF Multiphase Model." Numerical Heat Transfer 52 (2): 163-83.

[4] Hirt, C., and Nichols, B. 1975. "Volume of Fluid Method for Dynamic of Free Boundaries." Journal of Computational Physics 39 (1): 201-25.

[5] Wei, G. 2004. A Fixed Mesh Method for General Moving Objects in Fluid. New Mexico: Flow Science Inc.

[6] Barkudharov, M. 2004. Lagrangian VOF Advection Method for Flow-3D. New Mexico: Flow Science Inc.

[7] Youngs, D. L. 1982. Time-Dependent Multi-material Flow with Large Fluid Distortion. New York: Academic Press.

[8] Brackbill, J. U., Kothe, D. B., and Zema, C. 1992. "A Continuum Method for Modeling Surface Tension." Journal Computational Physics 100 (2): 335-54.

[9] Lee, R. J., DiGuilio, R. M., Jeter, S. M., and Teja, A. S. 1990. "Properties of Lithium Bromide Water Solution at High Temperatures and Concentartions Part II: Density and Viscosity." ASHRAE Trans. 96 (1): 704-28.

[10] Yao, W., Bjurström, H., and Oriolo, F. 1991. "Surface Tension of Lithium Bromide Solution with Heat-Transfer Addidives." Journal Chemical Engineering 36 (1): 96-8.

[11] Grossman, G. 1986. "Heat and Mass Transfer in Film 

Flow Confined by Wire Screen

Absorption." In Handbook of Heat and Mass Transfer. Vol. 2. Houston: Gulf Publishing Company, 211-57.

[12] Mve, H. O., Rullière, R., Goulet, R., and Haberschill, P. 2012. "Numerical and Experimental Analysis of a Flow Confined by a Wire Screen." In Proceedings of the International Conference of Numerical Analysis and Applied Mathematics, 70-3.
[13] Akhami, S., Zaleski, S., and Bussman, M. 2009. “A Mesh-Dependent Model for Applying Dynamic Contact Angles to Vof Simulation." Journal of Computational Physics 228 (15): 5370-89.

[14] Auro, A. S., and Sushanta, K. M. 2009. "Effect of Dynamic Contact Angle in a VOF (Volume of Fluid) Model for a Microfluidic Capillary Flow." Journal of Colloid and Interface Science 339 (2): 461-80. 